\title{
Hemolytic anemia in native valve infective endocarditis: a rare case report and review of literature.
}

\author{
Manju $^{1}$, V.D Dogra ${ }^{2}$, Dhiraj Kapoor ${ }^{3}$, Gaveshna $^{4}$, Gopal Singh $^{5}$ \\ ${ }^{1}$ M.D.Medicine, Senior Resident Department of Medicine Dr.RPGMC Tanda H.P. \\ ${ }^{2}$ Associate Professor, Department of Medicine Dr.RPGMC Tanda H.P. \\ ${ }^{3}$ Professor, Department of Medicine Dr.RPGMC Tanda H.P. \\ ${ }^{4}$ Assistant Professor, Department of Medicine Dr.RPGMC Tanda H.P. \\ ${ }^{5}$ M.D. Anaesthesia, Department of Anaesthesia, Zonal Hospital Dharamshala (H.P.) \\ DOI: https://doi.org/10.15520/ijmhs.v10i01.2787
}

Reviewed By: Dr

ABSTRACT

Denial V.

Department: Medical

Hemolytic anemia (HA) is not a common presentation in infectious endocarditis (IE) and has only been reported in very few cases. Although diseased valves may cause shearing stress that fragments RBCs, similar to that associated with mechanical heart valves, an autoimmune hemolytic process has also been implicated. We reported a case of patient who had HA secondary to acute IE due to MRSA. The anemia completely resolved after treating the (IE) . The cause was most likely mechanical shearing (schistocytes or fragmented RBCs present on peripheral smear) by the large vegetation on native valve ; autoimmune hemolysis was considered unlikely in this case due to consistently

*Email: drgopalsingh07@gmail.com. negative Coombs tests and failure to respond to corticosteroids.

\section{INTRODUCTION}

Mild normocytic anemia without reticulocytosis frequently accompanies conditions such as infective endocarditis, malignancy and chronic inflammations, and is generally recognized as the 'anemia of chronic disorder. ${ }^{1}$ Hemolytic anemia associated with native valve infective endocarditis has been noted only in a few previous reports. ${ }^{2}$ Here we report a case of severe debilitating HA occurring in an immunocompetent patient whose cause of anemia was only revealed after the discovery of acute bacterial endocarditis due to MRSA and a prolonged antibiotic treatment. We describe an unusual case of endocarditis-induced hemolysis that resolved after resolution of the vegetation on mitral valve.

\section{CASE REPORT}

A 19-year-old male was admitted in department medicine of tertiary care institution RPGMC Kangra at Tanda with a 20 days history of lowgrade fever ,intermittent type and documented 100 to $102^{0}$, headache, vomiting and pain abdomen . No significant past history. Not 
known case of diabetes mellitus, Hypertension, Valvular heart disease, no congenital heart disease. Not being treated for tuberculosis. He denied any history of intravenous drug abuse, sexual contact or family history of hematological disease. Progressive dyspnea, swelling and petechiae over the lower legs developed 2 days before admission. On admission, the patient was pale and febrile with a temperature of $102^{\circ} \mathrm{F}$, a heart rate of 108/min and a respiratory rate of 24 /min .On Physical Examination oral hygienic condition was good. Abdominal, cardiovascular and respiratory system were within normal limit. At that time, a complete blood cell count revealed mild leukocytosis (white blood cell count, $14.50 \times 103 / \mathrm{mm} 3)$ and anemia without CCF (hemoglobin $6 \mathrm{~g} / \mathrm{dl}$ ), platelet count, $210 \times 103 / \mathrm{mm} 3)$. The red cell distribution width was increased and the peripheral blood smear disclosed dimorphic anemia and few spherocytosis, schistocytes or polychromasia. His liver functions were deranged SGOT/SGPT 259/104.Mild elevation of unconjugated bilirubin was noted $(3.7 \mathrm{mg} / \mathrm{dl})$. Renal function remained normal throughout the course, Antinuclear antibody, anti-HIV antibody, sucrose hemolysis test, Ham's test and other examinations for abnormal hemoglobin were negative. The direct Coombs test was negative. Urine routine and microscopy was normal. Serology for scrub typhus, Leptospirosis, Malaria, Dengue and Typhoid was negative. Empirical treatment with broad spectrum antibiotic was given for 4-5 days. Symptomatically no improvement was seen and on repeat investigation, hemoglobin was $4.5 \mathrm{~g} / \mathrm{dl}$ and reticulocytes increased; lactate dehydrogenase was 846 U/L (normal: 47-140) and haptoglobin less than $24.3 \mathrm{mg} / \mathrm{dl}$ (normal, 53-170); the platelet count was $110 \times 103 / \mathrm{mm} 3$. A peripheral blood smears suggestive of few spherocytes, schistocytes and dimorphic anemia again. Glucose 6 PD was negative. Three sets Blood cuture was taken under aseptic condition and sent for culture sensitivity. B/C was positive for MRSA and sensitive for Vancomycin. Chest $\mathrm{X}$-rays and USG was also no significant. Echocardiography revealed native valve vegetation on mitral valve. Size of vegetation was $2 * 1.8 \mathrm{~cm}$. Modified Duke criteria was fulfilled and diagnosis of acute infective was made. Patient was treated with $\mathrm{I} / \mathrm{V}$ Vancomycine and ceftriaxone for 6 weeks. After Four weeks of IV antibiotics treatment; patient's HB was raised 10 $\mathrm{g} / \mathrm{dl}$, he became symptomatically better and his hemolysis also resolved.

\section{DISCUSSION}

Anemia is a common manifestation of infective endocarditis and is generally normochromic and normocytic but hemolytic anemia has been reported in only a few previous case reports ${ }^{1}$. A 2018 case report describes a patient with hypertrophic obstructive cardiomyopathy (HOCM) with left ventricular outflow tract (LVOT) obstruction who had HA secondary to sub-acute IE due to Actinomyces israelii. ${ }^{2}$ the anemia completely resolved after treating the IE. ${ }^{2}$ The cause was most likely mechanical shearing (schistocytes or fragmented RBCs present on peripheral smear) by the diseased valves; autoimmune hemolysis was considered unlikely in this case due to consistently negative Coombs tests and failure to respond to corticosteroids. ${ }^{2}$ Maeda et al reported a 65-yearold female with hemolysis associated with streptococcal endocarditis of the mitral valve. ${ }^{3}$ That patient had an underlying obstructive hypertrophic cardiomyopathy with a high pressure gradient in the left ventricular outflow tract and a moderate mitral regurgitation due to mitral valve prolapse. The infection improved after antibiotic treatment, but the hemolysis persisted. A reduction in the pressure gradient in the left ventricular outflow tract following propranolol administration resulted in an improvement of the hemolysis, probably due to a 
decrease in the shearing stress on the red blood cells. Nishiura et al described hemolytic anemia with Aspergillus endocarditis involving the mitral valve in a patient with acute lymphocytic leukemia. ${ }^{4}$ Postmortem examination of their patient showed huge vegetation on the mitral valve, and they postulated that the rapid progression of vegetations resulted in a powerful turbulent flow that caused intravascular hemolysis. Naidoo et al also reported intravascular hemolysis with fragmented erythrocytes in a patient with isolated endocarditis of the pulmonary valve. ${ }^{5}$ an underlying heart disease nor a specific microorganism was identified in that patient. The hematological abnormalities improved after 1 week of empiric antibiotic treatment. Inada et al also demonstrated improvement of hemolytic anemia after antibiotic therapy in a patient with Streptococcus sanguis endocarditis. ${ }^{6}$ In contrast, the patient with staphylococcal endocarditis involving the tricuspid valve due to drug abuse, reported by Gradon et al, required surgical removal of the vegetation for control of the hemolytic process. ${ }^{7}$

The presence of fragmented erythrocytes in all of these patients suggests that mechanical destruction of the red blood cells was responsible for the intravascular hemolysis. Obstruction of the right heart chamber by huge vegetations or obstruction in the high pressure system of the left heart may provide an adequate shearing stress on the erythrocytes leading to hemolysis. The reduction in shearing stress and loss of ability to produce enough antibodies due to the removal of the anti-genetic material resulted in the disappearance of the hemolytic anemia. We have described intravascular hemolysis in a patient without any congenital heart defects who had infective endocarditis with vegetations that involved the mitral valves. Hemolysis resolved after resolution of vegetation with antibiotic therapy. Infective endocarditis involving the native valve without under lying congenital heart defect and other predisposing condition like valvular heart disease, without cardiovascular implants and other imminocompromised conditions. Hemolytic anemia with left sided native valve vegetation and infective endocarditis with MRSA is rare. Most patients with a right and left -sided endocarditis have an underlying congenital heart disease such as ventricular septal defect, atrial septal defect, pulmonary stenosis, patent ductus arteriosus or hypertrophy obstructive cardiomyopathy.

\section{CONCLUSION}

This case highlights the importance of considering infectious causes i.e native valve infective endocarditis in the management of hemolytic anemia even in a patient who demonstrates no cardiac anatomical deformities. We describe an unusual case of endocarditisinduced hemolysis that resolved after resolution of the vegetation.

\section{REFERENCES}

1. Lee GR: The anemia of chronic disease. Semin Hematol 1983; 20: 61-80

2. Toom $\mathrm{S}, \mathrm{Xu}$ Y. Hemolytic anemia due to native valve subacute endocarditis with Actinomyces israellii infection. Clin Case Rep 2018;6: 376-79.

3. Maeda T, Ashie T, Kikuiri K, Fukuyama S, Yamaguchi Y, Yoshida E, et al: Fragmentation hemolysis in a patient with hypertrophic obstructive cardiomyopathy and mitral valve prolapse. Jpn Circ J 1992; 56: 970-74

4. Nishiura T, Miyazaki Y, Oritani K, Tominaga N, Tomiyama Y, Katagiri S, et al: Aspergillus vegetative endocarditis complicated with schizocytic hemolytic anemia in a patient with acute lymphocytic leukemia. Acta Haematol 1986; 76: 60-62 
5. Naidoo DP, Seedat MA, Vythilingum S: Isolated endocarditis of the pulmonary valve with fragmentation haemolysis. $\mathrm{Br}$ Heart J 1988; 60: 527-29

6. Inada T, Shirono K, Tsudo H: Hemolytic anemia in a patient with subacute bacterial endocarditis due to
Streptococcus sanguis. Acta Haematol 1995; 94: 95-97

7. Gradon JD, Hirschbein M, Milligan J: Fragmentation hemolysis: an unusual indication for valve replacement in native valve infective endocarditis. South Med J 1996; 89: 818-20 